This item was submitted to Loughborough's Research Repository by the author.

Items in Figshare are protected by copyright, with all rights reserved, unless otherwise indicated.

\title{
Green's functions for water waves in porous structures
}

PLEASE CITE THE PUBLISHED VERSION

LICENCE

CC BY-NC-ND 4.0

\section{REPOSITORY RECORD}

Linton, C.M., and P. Mclver. 2019. "Green's Functions for Water Waves in Porous Structures". figshare. https://hdl.handle.net/2134/809. 


\title{
GREEN'S FUNCTIONS FOR WATER WAVES IN POROUS STRUCTURES
}

\author{
by \\ C.M. Linton \& P. McIver
}

Department of Mathematical Sciences, Loughborough University, Leicestershire

LE11 3TU

\begin{abstract}
Representations for Green's functions suitable for water-wave problems involving porous structures are obtained by integrating solutions to appropriate heat conduction problems with respect to time. By utilizing different representations for these heat equation solutions for small and large times, the changeover being determined by an arbitrary positive parameter $a$, a one-parameter family of formulas for the required Green's function is derived and by varying $a$ the convergence characteristics of this new representation can be altered. Letting $a \rightarrow 0$ results in known eigenfunction expansions. The results of computations are presented showing the accuracy and efficiency of the resulting formulas.
\end{abstract}

\section{INTRODUCTION}

A number of mathematical models have been proposed to describe the interaction of water waves with permeable breakwaters. One class of models employs the theory of Sollitt and Cross (1972) which is an extension of the standard linearized theory of water waves. In the Sollitt and Cross theory, the whole flow field is described by a velocity potential that satisfies the Laplace equation. Within a surface-piercing permeable structure the free-surface condition has the same form as in the standard theory, but the frequency parameter is complex rather than real.

This theory has been applied by a number of authors. For example, Sulisz (1985) considered the two-dimensional problem of wave interaction with a permeable breakwater of arbitrary cross section. He employed a hybrid method in which the flow field within the constant-depth regions on either side of the breakwater was described using an eigenfunction expansion, and within and around the breakwater an integral-equation formulation based on an application of Green's theorem was used. The Green's function used was a simple source and therefore it was necessary to discretize all boundaries including the free surface.

In the two-dimensional problem, when the sides of the breakwater are vertical and the water depth is constant, it is possible to use an eigenfunction expansion throughout the 
fluid domain, including the interior of the breakwater. Indeed, this was done for normal incidence by Sollitt and Cross (1972) and subsequently extended to oblique wave incidence by Dalrymple, Losada, and Martin (1991). An application to breakwaters constructed from two layers of different material was made by Yu and Chwang (1994). The interaction of waves with a three-dimensional structure of arbitrary shape, but with vertical sides, was considered by Ijima, Chou, and Yumura (1974). They used an eigenfunction expansion in the vertical direction to reduce the full three-dimensional problem to an infinite sequence of two-dimensional problems which were solved using integral equations.

All of the works mentioned in the previous paragraph used vertical eigenfunctions satisfying the bed and free surface conditions. To construct these eigenfunctions, it is required to determine the solutions for $\mu$ of the dispersion relation

$$
K+\mu \tan \mu h=0,
$$

where $K$ is the specified frequency parameter and $h$ is the water depth. In the standard water-wave problem for time-harmonic waves, $K=\omega^{2} / g$ where $\omega$ is the angular frequency and $g$ is the acceleration due to gravity. Thus, in the standard problem, $K$ is real and it is known that there are two purely imaginary solutions and an infinity of purely real solutions for $\mu$. In each case, the zeros of a function of one real variable are required, and it is a straightforward procedure to determine these zeros numerically and hence construct the vertical eigenfunctions. Within a permeable structure, the Sollitt and Cross model gives $K=\omega^{2}(s+\mathrm{i} f) / g$ where $s>0$ is an inertia coefficient and $f>0$ is a friction coefficient. Thus $K$ is complex and the solution of (1) for the now complex roots $\mu$ is more difficult as it is required to find the zeros of a function of two real variables. Newton's method can be used to find these zeros provided a good initial guess in available. Dalrymple, Losada, and Martin (1991) describe a procedure based on Newton's method in which the roots for a particular complex $K$ are found by gradually increasing the imaginary part from zero. In fact, this procedure is unnecessary because it has been pointed out by McIver (1998) that explicit formulae are available for all of the solutions of (1), thus making the construction of the vertical eigenfunction relatively straightforward.

For structures with vertical sides, an alternative to the eigenfunction technique is to use integral equations based on a Green's function that satisfies both the free surface and bed conditions. When such a Green's function is used, it is necessary to discretize only the vertical sides of the breakwater. This approach has been validated by McLean (1999) for the problem solved by Dalrymple, Losada, and Martin (1991). A similar idea could also be used in three dimensions for breakwaters of arbitrary horizontal cross section.

McLean (1999) used an integral representation for the Green's function to avoid the need to compute the complex roots of (1). The integral representation has the disadvantage that it is very difficult to compute accurately when the source and field point are 
both very close to the free surface. However, Linton (1999), hereafter referred to as I, has recently devised a very efficient method for the computation of water-wave Green's functions that achieves high accuracy for all values of the parameters involved. The purpose of the present paper is to extend the work of Linton to complex $K$, and hence provide efficient and accurate Green's function algorithms for the breakwater problem. The method does require a small number (typically three to six) of the roots of (1), but the work of McIver (1998) (described in the Appendix) makes this straightforward.

We begin with the observation that solutions to Poisson's equation are related to solutions of the heat equation. Thus if

$$
\nabla^{2} \phi=f \quad \text { in } \Omega
$$

and

$$
\begin{aligned}
\nabla^{2} u & =u_{t} \\
u=-f & \text { in } \Omega, \\
u & \text { at } t=0,
\end{aligned}
$$

with $\phi$ and $u$ satisfying the same time-independent boundary conditions on $\partial \Omega$, then

$$
\phi=\int_{0}^{\infty} u \mathrm{~d} t
$$

provided this integral exists. If the integral does not exist we choose $\tilde{u}$ so that $\int_{0}^{\infty}(u+\tilde{u}) \mathrm{d} t$ does exist and then

$$
\phi=\int_{0}^{\infty}(u+\tilde{u}) \mathrm{d} t-\tilde{\phi}
$$

where

$$
\nabla^{2} \tilde{\phi}=-\left.\tilde{u}\right|_{t=0}
$$

If we can then find two complementary representations for $u+\tilde{u}, u_{1}$ and $u_{2}$, the first of which converges rapidly for small values of $t$, the latter being easily evaluated for large $t$, we can then introduce an arbitrary positive parameter $a$ and hence obtain a one-parameter family of formulas for $\phi$ in the form

$$
\phi=\int_{0}^{a} u_{1} \mathrm{~d} t+\int_{a}^{\infty} u_{2} \mathrm{~d} t-\tilde{\phi}
$$

These ideas were used by Strain (1992) to derive rapidly convergent series for the Green's function associated with Laplace's equation in an $n$-dimensional cube. 


\section{TWO HEAT CONDUCTION PROBLEMS}

In order to apply the method described above we need to solve certain heat conduction problems and it turns out that all the solutions we will need can be derived from two particular one-dimensional heat problems.

Consider first the problem corresponding to the release of a unit quantity of heat at $x=0$ at time $t=0$. In other words, taking the thermal diffusivity to be unity,

$$
\begin{array}{rlrl}
v_{x x} & =v_{t} & t>0,-\infty<x<\infty, \\
v & =\delta(x) & \text { at } t=0,
\end{array}
$$

where $\delta(x)$ is the Dirac delta function. It is easily verified that the solution is

$$
v=\frac{\mathrm{e}^{-x^{2} / 4 t}}{(4 \pi t)^{1 / 2}},
$$

for which $\int_{-\infty}^{\infty} v \mathrm{~d} x=1$ for all $t>0$.

Secondly we consider the problem

$$
\begin{array}{ll}
w_{z z}=w_{t} & t>0,-h<z \leq 0, \\
w_{z}=0 & \text { on } z=-h, \\
w_{z}=K w & \text { on } z=0, \\
w=\delta(z-\zeta) & \text { at } t=0,-h<\zeta \leq 0 .
\end{array}
$$

In terms of heat conduction, the boundary condition on $z=0$ is only physically realistic if $K$ is real and negative. In the water-wave problem considered in I, $K$ was taken to be a positive real constant, but here we will consider the case when $K$ is complex.

Our objective is to find two representations for the solution $w$ which converge rapidly for small $t$ and large $t$ respectively. First we separate out the singular behaviour of $w$ and write

$$
w=\frac{\mathrm{e}^{-(z-\zeta)^{2} / 4 t}}{(4 \pi t)^{1 / 2}}+w_{1}
$$

The method of Laplace transforms was used in I to show that

$$
\begin{aligned}
w & =-\frac{1}{2 \pi \mathrm{i}} \int_{c-\mathrm{i} \infty}^{c+\mathrm{i} \infty} \frac{\cosh q\left(z_{<}+h\right)\left(K \sinh q z_{>}+q \cosh q z_{>}\right)}{q(K \cosh q h-q \sinh q h)} \mathrm{e}^{p t} \mathrm{~d} p \\
& =\frac{\mathrm{e}^{-(z-\zeta)^{2} / 4 t}}{(4 \pi t)^{1 / 2}}+\frac{1}{2 \pi \mathrm{i}} \int_{c-\mathrm{i} \infty}^{c+\mathrm{i} \infty}\left[\mathrm{e}^{-q \chi_{0,4}}+\sum_{n=1}^{\infty}\left(\frac{q+K}{q-K}\right)^{n} \sum_{i=1}^{4} \mathrm{e}^{-q \chi_{n, i}}\right] \frac{\mathrm{e}^{p t}}{2 q} \mathrm{~d} p
\end{aligned}
$$

where $q^{2}=p$

$$
z_{<}=\min (z, \zeta), \quad z_{>}=\max (z, \zeta)
$$




$$
\begin{aligned}
& \chi_{n, 1}=2(n-1) h-\zeta-z, \\
& \chi_{n, 2}=2 n h-\zeta+z, \\
& \chi_{n, 3}=2 n h+\zeta-z, \\
& \chi_{n, 4}=2(n+1) h+\zeta+z,
\end{aligned}
$$

and $c$ is such that all the singularities of the integrand lie to the left of the line $(c-\mathrm{i} \infty, c+$ $\mathrm{i} \infty)$.

Since the integrand in (10) is an even function of $q=p^{1 / 2}$, there are no branch points in the complex $p$-plane. We write

$$
g(p)=K \cosh p^{\frac{1}{2}} h-p^{\frac{1}{2}} \sinh p^{\frac{1}{2}} h
$$

and this is zero when $p=-\mu^{2}$, where $\mu$ is a solution of the dispersion relation (1). From McIver (1998) we know that the roots of this equation are nearly always simple but that for some specific values of $K$ we may have a double root. We also know that if $\operatorname{Im}(K)>0$ then $\operatorname{Re}(\mu)>0$ and $\operatorname{Im}(\mu)<0$. The roots of the dispersion relation (1) will be labelled as follows. The simple poles are at $p=-\mu_{m}^{2}$ and $m$ begins from 1 if there is no pole for which $\operatorname{Re}\left(\mu^{2}\right) \leq 0$. If there is such a pole then we denote it $p=-\mu_{0}^{2}$. Numerical calculations suggest that there is at most one such pole, but we have been unable to prove this. In the event that more than one such pole can exist for a given complex $K$, the necessary modifications to the method below are straightforward. If there is a double pole we denote it by $p=-\tilde{\mu}^{2}$ and it follows from the work of McIver (1998) that there is at most one double pole. The argument principle can be used to count the number of poles in the sector $|\arg z| \leq \pi / 4,|z|<C$ for any constant $C$ and this can be used to show that any double pole must satisfy $\operatorname{Re}\left(\tilde{\mu}^{2}\right)>0$.

At a simple pole the integrand in (10) has a residue

$$
R_{m}=-\frac{\mathrm{e}^{-\mu_{m}^{2} t}}{N_{m}} \cos \mu_{m}(z+h) \cos \mu_{m}(\zeta+h),
$$

where

$$
N_{m}=\frac{h}{2}\left(1+\frac{\sin 2 \mu_{m} h}{2 \mu_{m} h}\right) .
$$

At a double pole, $p=-\tilde{\mu}^{2}$, we get a residue

$$
\tilde{R}=\frac{2 \mathrm{e}^{-\tilde{\mu}^{2} t}}{h \cos ^{2} \tilde{\mu} h}\left[\left(2 \tilde{\mu}^{2} t-1+\frac{4}{3} \sin ^{2} \tilde{\mu} h\right) Z^{(1)}+Z^{(2)}\right],
$$

where

$$
\begin{aligned}
& Z^{(1)}=\cos \tilde{\mu}(z+h) \cos \tilde{\mu}(\zeta+h) \\
& Z^{(2)}=\tilde{\mu}(\zeta+h) \cos \tilde{\mu}(z+h) \sin \tilde{\mu}(\zeta+h)+\tilde{\mu}(z+h) \cos \tilde{\mu}(\zeta+h) \sin \tilde{\mu}(z+h) .
\end{aligned}
$$


From (10), closing the contour in the left half plane, we thus obtain

$$
w=-\tilde{R}-\sum_{m=0}^{\infty} R_{m}
$$

where $\tilde{R}=0$ is there is no double root, and the sum starts from 1 if all the $\mu_{m}$ satisfy $\operatorname{Re}\left(\mu_{m}^{2}\right)>0$. The sum in (23) is rapidly convergent for large $t$.

An expression for $w$ that is rapidly convergent for small $t$ can be obtained by integrating (11) term by term exactly as in I. We obtain

$$
w=\frac{\mathrm{e}^{-(z-\zeta)^{2} / 4 t}}{(4 \pi t)^{1 / 2}}+\frac{\mathrm{e}^{-(2 h+\zeta+z)^{2} / 4 t}}{(4 \pi t)^{1 / 2}}+\sum_{m=1}^{\infty}(-1)^{m} \sum_{i=1}^{4} I_{m}\left(\chi_{m, i}\right),
$$

where the functions $I_{m}(\chi)$ are defined by the formula

$$
I_{m}(\chi)=\sum_{j=0}^{m} \frac{m !}{j !(m-j) !}(-1)^{j}(2 K)^{m-j} \tilde{I}_{m-j}(\chi)
$$

and the recurrence relation

$$
(n-1) \tilde{I}_{n}(\chi)=2 t \tilde{I}_{n-2}(\chi)+(\chi-2 K t) \tilde{I}_{n-1}(\chi),
$$

with the initial values

$$
\tilde{I}_{0}(\chi)=\frac{\mathrm{e}^{-\chi^{2} / 4 t}}{(4 \pi t)^{1 / 2}}, \quad \tilde{I}_{1}(\chi)=-\frac{1}{2} \mathrm{e}^{K^{2} t-K \chi} \operatorname{erfc}\left(\frac{\chi}{2 t^{1 / 2}}-K t^{1 / 2}\right) .
$$

In particular

$$
I_{0}(\chi)=\frac{\mathrm{e}^{-\chi^{2} / 4 t}}{(4 \pi t)^{1 / 2}}, \quad I_{1}(\chi)=-\frac{\mathrm{e}^{-\chi^{2} / 4 t}}{(4 \pi t)^{1 / 2}}-K \mathrm{e}^{K^{2} t-K \chi} \operatorname{erfc}\left(\frac{\chi}{2 t^{1 / 2}}-K t^{1 / 2}\right) .
$$

The series in (24) converges rapidly for small $t$ and we note that this representation is unaffected by the nature of the solutions to the dispersion relation (1). If we write $w_{N}$ for the solution to (5)-(8) with $K=0$, it was shown in I that

$$
w=w_{N}+\sum_{m=1}^{\infty} \sum_{j=0}^{\infty} \frac{(m+j) !}{m ! j !}(-2 K)^{m} \sum_{i=1}^{4} \tilde{I}_{m}\left(\chi_{m+j, i}\right) .
$$

\section{TWO-DIMENSIONAL GREEN'S FUNCTIONS}

We consider the two-dimensional fluid domain $-\infty<x<\infty,-h<z \leq 0$ with the undisturbed free surface being $z=0$ so that the Green's function representing an oscillating point source at $x=0, z=\zeta$ is $\operatorname{Re}\left\{G \mathrm{e}^{-\mathrm{i} \omega t}\right\}$ (this $t$ is of course unrelated to the $t$ of $\S 2$ and that which appears following (36) below) where $G$ is the solution to

$$
\begin{aligned}
\nabla_{x z}^{2} G & =\delta(x) \delta(z-\zeta) & & -h<z \leq 0,-h<\zeta \leq 0, \\
G_{z} & =K G & & \text { on } z=0, \\
G_{z} & =0 & & \text { on } z=-h,
\end{aligned}
$$


and we require $G$ to decay to zero as $|x| \rightarrow \infty$. Here $K=\omega^{2}(s+\mathrm{i} f) / g$ is a complex number for which both the real and imaginary parts are positive. As $f \rightarrow 0$ with $s=1$ this Green's function should go over to the standard free-surface water-wave Green's function, which behaves like outgoing waves as $|x| \rightarrow \infty$. The following definitions will be used:

$$
\rho=\left[x^{2}+(z-\zeta)^{2}\right]^{1 / 2}, \quad \rho^{\prime}=\left[x^{2}+(2 h+z+\zeta)^{2}\right]^{1 / 2} .
$$

Various representations exist for this Green's function. For example

$$
G=-\frac{1}{\pi} \int_{0}^{\infty} \frac{K \sinh k z_{>}+k \cosh k z_{>}}{k \sinh k h-K \cosh k h} \cosh k\left(z_{<}+h\right) \cos k x \frac{\mathrm{d} k}{k},
$$

where $z_{<}$and $z_{>}$are defined in (12), and this can be converted into an eigenfunction expansion as described in Dalrymple, Losada, and Martin (1991). If all the poles of the integrand in (33) are simple we obtain

$$
G=-\sum_{m=0}^{\infty} \frac{\cos \mu_{m}(z+h) \cos \mu_{m}(\zeta+h)}{2 \mu_{m} N_{m}} \mathrm{e}^{-\mu_{m}|x|},
$$

but if there is a double pole at $k=\tilde{\mu}$, we get an extra contribution

$$
\frac{\mathrm{e}^{-\tilde{\mu} x}}{\tilde{\mu} h \cos ^{2} \tilde{\mu} h}\left[\left(\tilde{\mu}|x|+\frac{4}{3} \sin ^{2} \tilde{\mu} h\right) Z^{(1)}+Z^{(2)}\right] .
$$

The integral (33) can be difficult to evaluate numerically, particularly when $|z|$ and $|\zeta|$ are both small. The eigenfunction series (34) converges very slowly if $|x|$ is small.

The method described in the introduction shows that in order to derive a new representation for $G$ we require the solution to the initial boundary-value problem

$$
\begin{aligned}
\nabla_{x z}^{2} u & =u_{t} & & t>0,-h<z \leq 0, \\
u_{z} & =K u & & \text { on } z=0, \\
u_{z} & =0 & & \text { on } z=-h, \\
u & =-\delta(x) \delta(z-\zeta) & & \text { at } t=0,-h<\zeta \leq 0 .
\end{aligned}
$$

From the results in $\S 2$ and the fact that the solution of the two-dimensional problem (36)-(39) can be written as a product of solutions to two one-dimensional problems (see Carslaw and Jaeger 1959, §1.15), this is simply

$$
u(x, z, t)=-\frac{\mathrm{e}^{-x^{2} / 4 t}}{(4 \pi t)^{1 / 2}} w(z, t),
$$

where $w$ is given by (23) or by (24), the two representations converging rapidly for large $t$ and small $t$ respectively.

First we will assume that there is no double root of the dispersion relation. If there is a pole for which $\operatorname{Re}\left(\mu^{2}\right) \leq 0$ (labelled $\mu_{0}$ ), then $\int_{0}^{\infty} u \mathrm{~d} t$ does not exist, so we consider the function $u+\tilde{u}$, where

$$
\tilde{u}=\frac{\mathrm{e}^{-x^{2} / 4 t-\mu_{0}^{2} t}}{(4 \pi t)^{1 / 2} N_{0}} \cos \mu_{0}(z+h) \cos \mu_{0}(\zeta+h) .
$$


which satisfies the initial condition

$$
\tilde{u}=N_{0}^{-1} \delta(x) \cos \mu_{0}(z+h) \cos \mu_{0}(\zeta+h) \quad \text { at } t=0
$$

and hence, since $\left(\mathrm{d}^{2} / \mathrm{d} x^{2}-\mu_{0}^{2}\right) \exp \left(-\mu_{0}|x|\right)=-2 \mu_{0} \delta(x)$,

$$
\int_{0}^{\infty}(u+\tilde{u}) \mathrm{d} t=\frac{\mathrm{e}^{-\mu_{0}|x|}}{2 \mu_{0} N_{0}} \cos \mu_{0}(z+h) \cos \mu_{0}(\zeta+h)+G .
$$

In order to obtain exponentially convergent representations for $G$ we introduce an arbitrary positive parameter $a$, split the range of integration at $a^{2} h^{2} / 4$ and use the appropriate form for $u+\tilde{u}$ in each part of the integral. We obtain

$$
\begin{aligned}
G= & -\frac{\mathrm{e}^{-\mu_{0}|x|}}{2 \mu_{0} N_{0}} \cos \mu_{0}(z+h) \cos \mu_{0}(\zeta+h)-\sum_{m=0}^{\infty} \frac{\Lambda_{m}}{N_{m}} \cos \mu_{m}(z+h) \cos \mu_{m}(\zeta+h) \\
& -\frac{1}{4 \pi} E_{1}\left(\frac{\rho^{2}}{a^{2} h^{2}}\right)-\frac{1}{4 \pi} E_{1}\left(\frac{\rho^{\prime 2}}{a^{2} h^{2}}\right)-\sum_{n=1}^{\infty}(-1)^{n} L_{n},
\end{aligned}
$$

where $E_{1}(\cdot)$ is an exponential integral,

$$
\begin{aligned}
\Lambda_{0} & =-\int_{0}^{a^{2} h^{2} / 4} \frac{\mathrm{e}^{-x^{2} / 4 t}}{(4 \pi t)^{1 / 2}} \mathrm{e}^{-\mu_{0}^{2} t} \mathrm{~d} t \\
\Lambda_{m} & =\int_{a^{2} h^{2} / 4}^{\infty} \frac{\mathrm{e}^{-x^{2} / 4 t}}{(4 \pi t)^{1 / 2}} \mathrm{e}^{-\mu_{m}^{2} t} \mathrm{~d} t
\end{aligned}
$$

and

$$
L_{n}=\int_{0}^{a^{2} h^{2} / 4} \frac{\mathrm{e}^{-x^{2} / 4 t}}{(4 \pi t)^{1 / 2}} \sum_{i=1}^{4} I_{n}\left(\chi_{n, i}\right) \mathrm{d} t
$$

If we let $a \rightarrow 0$ in (44) we recover the eigenfunction expansion (34).

It is straightforward to evaluate the integrals $\Lambda_{m}$ numerically since (with the exception of $\Lambda_{0}$ when $x=0$ ) the integrands decay exponentially. In fact the integrals in (46) and (47) can be written in terms of error functions. From Abramowitz and Stegun (1965), eqn 7.4.33, we can show that

$$
\begin{aligned}
\Lambda_{0} & =-\frac{1}{4 \mu_{0}}\left[\mathrm{e}^{\mu_{0}|x|} \operatorname{erf}\left(\frac{\mu_{0} a h}{2}+\frac{|x|}{a h}\right)+\mathrm{e}^{-\mu_{0}|x|} \operatorname{erf}\left(\frac{\mu_{0} a h}{2}-\frac{|x|}{a h}\right)-2 \sinh \left(\mu_{0}|x|\right)\right], \\
\Lambda_{m} & =\frac{1}{4 \mu_{m}}\left[\mathrm{e}^{\mu_{m}|x|} \operatorname{erfc}\left(\frac{\mu_{m} a h}{2}+\frac{|x|}{a h}\right)+\mathrm{e}^{-\mu_{m}|x|} \operatorname{erfc}\left(\frac{\mu_{m} a h}{2}-\frac{|x|}{a h}\right)\right],
\end{aligned}
$$

which simplify to

$$
\Lambda_{0}=-\frac{1}{2 \mu_{0}} \operatorname{erf}\left(\frac{\mu_{0} a h}{2}\right), \quad \Lambda_{m}=\frac{1}{2 \mu_{m}} \operatorname{erfc}\left(\frac{\mu_{m} a h}{2}\right),
$$

when $x=0$. Chaudhry, Temme, and Veling (1996) give a number of series expansions which can also be used to compute $\Lambda_{m}$. 
The integrals $L_{n}$ must be evaluated numerically but, as will be demonstrated below, provided $a$ is small enough, only $L_{1}$ is required. From (28) we have that

$$
L_{1}=-\frac{1}{4 \pi} \sum_{i=1}^{4} E_{1}\left(\frac{x^{2}+\chi_{1, i}^{2}}{a^{2} h^{2}}\right)-\frac{K}{\pi^{1 / 2}} \int_{0}^{a h / 2} \mathrm{e}^{K^{2} u^{2}-x^{2} / 4 u^{2}} \sum_{i=1}^{4} \mathrm{e}^{-K \chi_{1, i}} \operatorname{erfc}\left(\frac{\chi_{1, i}}{2 u}-K u\right) \mathrm{d} u
$$

and, from (25)-(27), we can show that

$$
\begin{aligned}
L_{2}= & \frac{1}{4 \pi} \sum_{i=1}^{4} E_{1}\left(\frac{x^{2}+\chi_{2, i}^{2}}{a^{2} h^{2}}\right)+\frac{K^{2} a^{2} h^{2}}{2 \pi} \sum_{i=1}^{4} E_{2}\left(\frac{x^{2}+\chi_{2, i}^{2}}{a^{2} h^{2}}\right) \\
& -\frac{2 K}{\pi^{1 / 2}} \int_{0}^{a h / 2} \mathrm{e}^{K^{2} u^{2}-x^{2} / 4 u^{2}} \sum_{i=1}^{4}\left(K \chi_{2, i}-2 K^{2} u^{2}-1\right) \mathrm{e}^{-K \chi_{2, i}} \operatorname{erfc}\left(\frac{\chi_{2, i}}{2 u}-K u\right) \mathrm{d} u .
\end{aligned}
$$

If we introduce the truncation parameter $M$ and ignore the terms $L_{n}, n \geq 2$, in (44), we have the formula

$$
\begin{aligned}
G= & -\frac{\mathrm{e}^{-\mu_{0}|x|}}{2 \mu_{0} N_{0}} \cos \mu_{0}(z+h) \cos \mu_{0}(\zeta+h)-\sum_{m=0}^{M} \frac{\Lambda_{m}}{N_{m}} \cos \mu_{m}(z+h) \cos \mu_{m}(\zeta+h) \\
& -\frac{1}{4 \pi}\left[E_{1}\left(\frac{\rho^{2}}{a^{2} h^{2}}\right)+E_{1}\left(\frac{\rho^{\prime 2}}{a^{2} h^{2}}\right)+\sum_{i=1}^{4} E_{1}\left(\frac{x^{2}+\chi_{1, i}^{2}}{a^{2} h^{2}}\right)\right] \\
& -\frac{K}{\pi^{1 / 2}} \int_{0}^{a h / 2} \mathrm{e}^{K^{2} u^{2}-x^{2} / 4 u^{2}} \sum_{i=1}^{4} \mathrm{e}^{-K \chi_{1, i}} \operatorname{erfc}\left(\frac{\chi_{1, i}}{2 u}-K u\right) \mathrm{d} u+\ldots,
\end{aligned}
$$

where $\Lambda_{0}$ is given by (45) and $\Lambda_{m}, m \geq 1$, by (46). If there is no pole for which $\operatorname{Re}\left(\mu^{2}\right) \leq 0$, the terms containing $\mu_{0}$ are simply removed from this formula. If there is a double root the only change is that we must add a term

$$
D=\int_{a^{2} h^{2} / 4}^{\infty} \frac{\mathrm{e}^{-x^{2} / 4 t}}{(4 \pi t)^{1 / 2}} \tilde{R} \mathrm{~d} t
$$

to (53), where $\tilde{R}$ is given by (20). It follows that

$$
D=\frac{2}{h \cos ^{2} \tilde{\mu} h}\left[2 \tilde{\mu}^{2} Q_{1} Z^{(1)}+Q_{2}\left(\left(-1+\frac{4}{3} \sin ^{2} \tilde{\mu} h\right) Z^{(1)}+Z^{(2)}\right)\right],
$$

where, see Chaudhry, Temme, and Veling (1996),

$$
\begin{aligned}
Q_{1}= & \int_{a^{2} h^{2} / 4}^{\infty} \frac{t^{1 / 2} \mathrm{e}^{-x^{2} / 4 t-\tilde{\mu}^{2} t}}{(4 \pi)^{1 / 2}} \mathrm{~d} t \\
= & \frac{1}{8 \tilde{\mu}^{2}}\left[\mathrm{e}^{\tilde{\mu}|x|}\left(\frac{1}{\mu}-|x|\right) \operatorname{erfc}\left(\frac{\tilde{\mu} a h}{2}+\frac{|x|}{a h}\right)+\mathrm{e}^{-\tilde{\mu}|x|}\left(\frac{1}{\mu}+|x|\right) \operatorname{erfc}\left(\frac{\tilde{\mu} a h}{2}-\frac{|x|}{a h}\right)\right] \\
& \quad+\frac{a h}{4 \tilde{\mu}^{2} \pi^{1 / 2}} \exp \left(-\frac{\tilde{\mu}^{2} a^{2} h^{2}}{4}-\frac{x^{2}}{a^{2} h^{2}}\right) \\
Q_{2}= & \int_{a^{2} h^{2} / 4}^{\infty} \frac{\mathrm{e}^{-x^{2} / 4 t-\tilde{\mu}^{2} t}}{(4 \pi t)^{1 / 2}} \mathrm{~d} t \\
= & \frac{1}{4 \tilde{\mu}}\left[\mathrm{e}^{\tilde{\mu}|x|} \operatorname{erfc}\left(\frac{\tilde{\mu} a h}{2}+\frac{|x|}{a h}\right)+\mathrm{e}^{-\tilde{\mu}|x|} \operatorname{erfc}\left(\frac{\tilde{\mu} a h}{2}-\frac{|x|}{a h}\right)\right]
\end{aligned}
$$


When $x=0$ the extra contribution due to the double pole is simply

$$
D=\frac{1}{\tilde{\mu} h \cos ^{2} \tilde{\mu} h}\left[\tilde{\mu} a h \pi^{-\frac{1}{2}} Z^{(1)} \mathrm{e}^{-\tilde{\mu}^{2} a^{2} h^{2} / 4}+\left(\frac{4}{3} Z^{(1)} \sin ^{2} \tilde{\mu} h+Z^{(2)}\right) \operatorname{erfc}\left(\frac{\tilde{\mu} a h}{2}\right)\right] .
$$

An alternative expression for the Green's function can be obtained by writing

$$
G=G_{N}+G_{K}
$$

where $G_{N}$ satisfies Neumann boundary conditions on $z=-h$ and $z=0$. The function $G_{N}$ can be written in closed form:

$$
G_{N}=\frac{1}{2 \pi} \ln \left(2\left|\cos \frac{\pi \zeta}{h}-\cos \frac{\pi}{h}(z+\mathrm{i} x)\right|\right) .
$$

The method used to derive (44) can now be used to obtain a rapidly convergent expansion for $G_{K}$. Instead of (41) we use

$$
u+\tilde{u}=-\frac{\mathrm{e}^{-x^{2} / 4 t}}{(4 \pi t)^{1 / 2}}\left(w-w_{N}-\frac{\mathrm{e}^{-\mu_{0}^{2} t}}{N_{0}} \cos \mu_{0}(z+h) \cos \mu_{0}(\zeta+h)+\frac{1}{h}\right),
$$

where $w$ is the solution to (5)-(8) and $w_{N}$ is the solution to the same boundary-value problem with $K=0$. A formula for $w-w_{N}$ suitable for large times is, from (23),

$$
w-w_{N}=\tilde{R}+\sum_{m=0}^{\infty}\left[R_{m}-\frac{\epsilon_{m}}{h} \mathrm{e}^{-m^{2} \pi^{2} t / h^{2}} \cos \frac{m \pi z}{h} \cos \frac{m \pi \zeta}{h}\right],
$$

where $\epsilon_{0}=1, \epsilon_{m}=2, m \geq 1$, and a form for $w-w_{N}$ which converges rapidly for small times can be obtained from (29). Now, at $t=0$,

$$
u+\tilde{u}=N_{0}^{-1} \delta(x) \cos \mu_{0}(z+h) \cos \mu_{0}(\zeta+h)-h^{-1} \delta(x)-\delta(x) \delta(z-\zeta),
$$

and hence

$$
\int_{0}^{\infty}(u+\tilde{u}) \mathrm{d} t=\frac{\mathrm{e}^{-\mu_{0}|x|}}{2 \mu_{0} N_{0}} \cos \mu_{0}(z+h) \cos \mu_{0}(\zeta+h)+\frac{|x|}{2 h}+G_{K} .
$$

Following the same procedure as before yields

$$
\begin{aligned}
G_{K}= & -\frac{\mathrm{e}^{-\mu_{0}|x|}}{2 \mu_{0} N_{0}} \cos \mu_{0}(z+h) \cos \mu_{0}(\zeta+h)-\frac{|x|}{2 h}-\sum_{m=1}^{\infty} \sum_{j=0}^{\infty} \frac{(m+j) !}{m ! j !}(-2 K)^{m} L_{m j} \\
& -\sum_{m=0}^{\infty}\left[\frac{\Lambda_{m}}{N_{m}} \cos \mu_{m}(z+h) \cos \mu_{m}(\zeta+h)-\frac{\epsilon_{m}}{h} M_{m} \cos \frac{m \pi z}{h} \cos \frac{m \pi \zeta}{h}\right],
\end{aligned}
$$

where $\Lambda_{m}$ is given by (45) and (46),

$$
\begin{aligned}
M_{0} & =-\int_{0}^{a^{2} h^{2} / 4} \frac{\mathrm{e}^{-x^{2} / 4 t}}{(4 \pi t)^{1 / 2}} \mathrm{~d} t=-\frac{|x|}{4 \pi^{1 / 2}} \Gamma\left(-\frac{1}{2}, \frac{x^{2}}{a^{2} h^{2}}\right), \\
M_{m} & =\int_{a^{2} h^{2} / 4}^{\infty} \frac{\mathrm{e}^{-x^{2} / 4 t}}{(4 \pi t)^{1 / 2}} \mathrm{e}^{-m^{2} \pi^{2} t / h^{2}} \mathrm{~d} t \\
& =\frac{h}{4 m \pi}\left[\mathrm{e}^{m \pi|x| / h} \operatorname{erfc}\left(\frac{m \pi a}{2}+\frac{|x|}{a h}\right)+\mathrm{e}^{-m \pi|x| / h} \operatorname{erfc}\left(\frac{m \pi a}{2}-\frac{|x|}{a h}\right)\right],
\end{aligned}
$$


and

$$
L_{m j}=\int_{0}^{a^{2} h^{2} / 4} \frac{\mathrm{e}^{-x^{2} / 4 t}}{(4 \pi t)^{1 / 2}} \sum_{i=1}^{4} \tilde{I}_{m}\left(\chi_{m+j, i}\right) \mathrm{d} t .
$$

In particular

$$
L_{10}=-\frac{1}{2 \pi^{1 / 2}} \int_{0}^{a h / 2} \mathrm{e}^{K^{2} u^{2}-x^{2} / 4 u^{2}} \sum_{i=1}^{4} \mathrm{e}^{-K \chi_{1, i}} \operatorname{erfc}\left(\frac{\chi_{1, i}}{2 u}-K u\right) \mathrm{d} u
$$

and we note that when $x=0$,

$$
M_{0}=-\frac{a h}{2 \pi^{1 / 2}}, \quad M_{m}=\frac{h}{2 m \pi} \operatorname{erfc}\left(\frac{m \pi a}{2}\right) .
$$

From (59), (60) and (65) we have, neglecting the all terms $L_{m j}$ except $L_{10}$,

$$
\begin{aligned}
G= & -\frac{|x|}{2 h}+\frac{1}{2 \pi} \ln \left(2\left|\cos \frac{\pi \zeta}{h}-\cos \frac{\pi}{h}(z+\mathrm{i} x)\right|\right)-\frac{\mathrm{e}^{-\mu_{0}|x|}}{2 \mu_{0} N_{0}} \cos \mu_{0}(z+h) \cos \mu_{0}(\zeta+h) \\
& -\sum_{m=0}^{\infty}\left[\frac{\Lambda_{m}}{N_{m}} \cos \mu_{m}(z+h) \cos \mu_{m}(\zeta+h)-\frac{\epsilon_{m}}{h} M_{m} \cos \frac{m \pi z}{h} \cos \frac{m \pi \zeta}{h}\right] \\
& -\frac{K}{\pi^{1 / 2}} \int_{0}^{a h / 2} \mathrm{e}^{K^{2} u^{2}-x^{2} / 4 u^{2}} \sum_{i=1}^{4} \mathrm{e}^{-K \chi_{1, i}} \operatorname{erfc}\left(\frac{\chi_{1, i}}{2 u}-K u\right) \mathrm{d} u+\ldots
\end{aligned}
$$

If there is a double pole we must add a term $D$ given by (55) and if there is no pole for which $\operatorname{Re}\left(\mu^{2}\right) \leq 0$, the terms containing $\mu_{0}$ are simply removed from this formula; exactly as before.

\section{THREE-DIMENSIONAL GREEN'S FUNCTIONS}

Next we consider the three-dimensional fluid domain $-\infty<x<\infty,-\infty<y<\infty$, $-h<z \leq 0$ with the undisturbed free surface being $z=0$ so that the Green's function representing a point source at $x=0, z=\zeta$, again factoring out a time dependence of $\exp (-\mathrm{i} \omega t)$, is the solution to

$$
\nabla^{2} G=\delta(x) \delta(y) \delta(z-\zeta) \quad-h<z \leq 0,-h<\zeta \leq 0
$$

together with (31) and (32), and we require $G$ to tend to zero as $R=\left(x^{2}+y^{2}\right)^{\frac{1}{2}} \rightarrow \infty$. The following definitions will be used:

$$
r=\left[x^{2}+y^{2}+(z-\zeta)^{2}\right]^{\frac{1}{2}}, \quad r^{\prime}=\left[x^{2}+y^{2}+(2 h+z+\zeta)^{2}\right]^{\frac{1}{2}} .
$$

As in the two-dimensional case we can represent $G$ as an integral or as an eigenfunction expansion:

$$
\begin{aligned}
G & =-\frac{1}{2 \pi} \int_{0}^{\infty} \frac{K \sinh k z_{>}+k \cosh k z_{>}}{k \sinh k h-K \cosh k h} \cosh k\left(z_{<}+h\right) J_{0}(k R) \mathrm{d} k \\
& =-\sum_{m=0}^{\infty} \frac{K_{0}\left(\mu_{m} R\right)}{2 \pi N_{m}} \cos \mu_{m}(z+h) \cos \mu_{m}(\zeta+h) .
\end{aligned}
$$


Again, direct numerical integration is difficult when $|z|$ and $|\zeta|$ are both small and evaluation of the eigenfunction sum is difficult when $|R|$ is small.

To derive a new representation for $G$ we require the solution to the initial boundaryvalue problem

$$
\begin{aligned}
\nabla^{2} u & =u_{t} & & t>0,-h<z \leq 0, \\
u & =-\delta(x) \delta(y) \delta(z-\zeta) & & \text { at } t=0,-h<\zeta \leq 0 .
\end{aligned}
$$

together with (37) and (38). This is simply

$$
u(x, y, z, t)=-\frac{\mathrm{e}^{-R^{2} / 4 t}}{4 \pi t} w(z, t),
$$

where $w$ is given by $(23)$ or by $(24)$.

Once again $\int_{0}^{\infty} u \mathrm{~d} t$ does not exist if there is a pole for which $\operatorname{Re}\left(\mu^{2}\right) \leq 0$, so instead we consider the function

$$
u+\tilde{u}=-\frac{\mathrm{e}^{-R^{2} / 4 t}}{4 \pi t}\left[w-\frac{\mathrm{e}^{-\mu_{0}^{2} t}}{N_{0}} \cos \mu_{0}(z+h) \cos \mu_{0}(\zeta+h)\right] .
$$

so that $\tilde{u}$ satisfies the initial condition

$$
\tilde{u}=N_{0}^{-1} \delta(x) \delta(y) \cos \mu_{0}(z+h) \cos \mu_{0}(\zeta+h) \quad \text { at } t=0,
$$

and hence,

$$
\int_{0}^{\infty}(u+\tilde{u}) \mathrm{d} t=\frac{K_{0}\left(\mu_{0} R\right)}{2 \pi N_{0}} \cos \mu_{0}(z+h) \cos \mu_{0}(\zeta+h)+G .
$$

Following the same procedure as in the two-dimensional case we obtain, introducing a truncation parameter $M$,

$$
\begin{aligned}
G= & -\sum_{m=0}^{M} \frac{\Lambda_{m}}{N_{m}} \cos \mu_{m}(z+h) \cos \mu_{m}(\zeta+h) \\
& -\frac{1}{4 \pi r} \operatorname{erfc}\left(\frac{r}{a h}\right)-\frac{1}{4 \pi r^{\prime}} \operatorname{erfc}\left(\frac{r^{\prime}}{a h}\right)-\sum_{i=1}^{4} \frac{\operatorname{erfc}\left[(a h)^{-1}\left(R^{2}+\chi_{1, i}^{2}\right)^{1 / 2}\right]}{4 \pi\left(R^{2}+\chi_{1, i}^{2}\right)^{1 / 2}} \\
& -\frac{K}{2 \pi} \int_{0}^{a h / 2} \mathrm{e}^{K^{2} u^{2}-R^{2} / 4 u^{2}} \sum_{i=1}^{4} \mathrm{e}^{-K \chi_{1, i}} \operatorname{erfc}\left(\frac{\chi_{1, i}}{2 u}-K u\right) \frac{\mathrm{d} u}{u}+\ldots,
\end{aligned}
$$

where now

$$
\begin{aligned}
\Lambda_{0} & =\frac{K_{0}\left(\mu_{0} R\right)}{2 \pi}-\int_{0}^{a^{2} h^{2} / 4} \frac{\mathrm{e}^{-R^{2} / 4 t}}{4 \pi t} \mathrm{e}^{-\mu_{0}^{2} t} \mathrm{~d} t, \\
\Lambda_{m} & =\int_{a^{2} h^{2} / 4}^{\infty} \frac{\mathrm{e}^{-R^{2} / 4 t}}{4 \pi t} \mathrm{e}^{-\mu_{m}^{2} t} \mathrm{~d} t .
\end{aligned}
$$

If there is no pole for which $\operatorname{Re}\left(\mu^{2}\right) \leq 0$, the terms containing $\mu_{0}$ are removed. If there is a double root we must add a term

$$
D=\int_{a^{2} h^{2} / 4}^{\infty} \frac{\mathrm{e}^{-R^{2} / 4 t}}{4 \pi t} \tilde{R} \mathrm{~d} t
$$


to (81), where $\tilde{R}$ is given by (20). This integral is evaluated numerically except when $R=0$ in which case the extra contribution due to the double pole is simply

$$
D=\frac{1}{\pi h \cos ^{2} \tilde{\mu} h}\left[Z^{(1)} \mathrm{e}^{-\tilde{\mu}^{2} a^{2} h^{2} / 4}+\frac{1}{2} E_{1}\left(\frac{\tilde{\mu}^{2} a^{2} h^{2}}{4}\right)\left[\left(-1+\frac{4}{3} \sin ^{2} \tilde{\mu} h\right) Z^{(1)}+Z^{(2)}\right]\right] .
$$

If we let $a \rightarrow 0$ in (81) we recover the eigenfunction expansion (74). By letting $a \rightarrow 0$ in (84) we find that the additional contribution to the eigenfunction expansion due to the double pole is

$$
\frac{1}{\pi h \cos ^{2} \tilde{\mu} h}\left[Z^{(1)} \tilde{\mu} R K_{1}(\tilde{\mu} R)+\left[\left(-1+\frac{4}{3} \sin ^{2} \tilde{\mu} h\right) Z^{(1)}+Z^{(2)}\right] K_{0}(\tilde{\mu} R)\right] .
$$

This is the three-dimensional equivalent of the expression (35) derived by Dalrymple, Losada, and Martin (1991) and, as far as the authors are aware, has not been derived previously.

The expression (82) is not suitable for the numerical evaluation of $\Lambda_{0}$ when $R$ is small. If we expand $K_{0}\left(\mu_{0} R\right)$ for small $R$ and expand $\mathrm{e}^{-\mu_{0}^{2} t}$ in a power series we can show that

$$
\begin{aligned}
-4 \pi \Lambda_{0}= & 2 \ln \frac{\mu_{0} a h}{2}+2 \sum_{n=1}^{\infty} \frac{\left(\mu_{0} R\right)^{2 n}}{2^{2 n}(n !)^{2}}\left[\ln \frac{\mu_{0} R}{2}-\psi(n+1)\right] \\
& +\gamma+\sum_{n=1}^{\infty} \frac{(-1)^{n}}{n !}\left[\left(\frac{\mu_{0} a h}{2}\right)^{2 n} E_{n+1}\left(\frac{R^{2}}{a^{2} h^{2}}\right)-\frac{1}{n}\left(\frac{R}{a h}\right)^{2 n}\right],
\end{aligned}
$$

where $\gamma$ is Euler's constant and $\psi(\cdot)$ is the Digamma function. To evaluate the limit as $R \rightarrow 0$ of the right-hand side of (86) we note that for $m \geq 2, E_{m}(0)=1 /(m-1)$ and use Abramowitz and Stegun (1965), eqn 5.1.11. We obtain

$$
\lim _{R \rightarrow 0} \Lambda_{0}=\frac{1}{4 \pi} E_{1}\left(\frac{\mu_{0}^{2} a^{2} h^{2}}{4}\right)
$$

and, directly from (83),

$$
\left.\Lambda_{m}\right|_{R=0}=\frac{1}{4 \pi} E_{1}\left(\frac{\mu_{m}^{2} a^{2} h^{2}}{4}\right)
$$

\section{RESULTS}

In this section, the value of $h$ has, without loss of generality, been fixed at unity. The value of $K$ with the smallest modulus for which a double root occurs will be labelled $K_{d}$ and to 12 decimal places $K_{d}=1.650611293540+2.059981457180 \mathrm{i}$. All the numerical computations below were performed using MathematicA.

There are a number of considerations which effect the choice of algorithm for the evaluation of $G$ in particular application. Many of the formulas listed in this paper involve special functions and so if these are to be used, efficient algorithms for the computation 
of these functions must be available. A survey of algorithms available for the evaluation of special functions is provided by Lozier and Olver (1995). Below we will concentrate on determining the number of terms in each series that are required to achieve an accuracy of 8 decimal places in both the real and imaginary parts of $G$. Whilst this accuracy will be adequate for most practical applications it should be noted that, because of the exponential convergence rate of the representations for $G$ derived in this paper, very little extra effort is required to increase this to 10 or 12 decimal places of accuracy. In many applications it is the spatial derivatives of $G$ that are required; these can be obtained by differentiating the formulas in this paper term by term and the resulting expressions will converge slower than those for $G$. Typically this process introduces an algebraically growing factor which multiplies the exponentially decaying factor. As a result one can usually expect 1 or 2 decimal places less accuracy for the same truncation parameters.

We will not address the question of the relative CPU usage of the various methods however, except with the following general remarks. Our experience shows that, except when $|z|$ and $|\zeta|$ are both small, the integral representation is the quickest method, not least because there is no need to compute any of the roots to the dispersion relation. The situation is better for the case of complex $K$ than for the standard water-wave case, for which $K$ is real, because in the latter case there is a pole of the integrand on the real axis which necessitates the evaluation of a principal-value integral. However for small $|z|$ and $|\zeta|$ the integral converges very slowly and the representation is not useful. Just when the integral representation can be used efficiently is hard to determine. For large $|x|$ ( $R$ in three dimensions) the eigenfunction expansion converges quickly and only a few of the roots to the dispersion relation are required. However as $|x|$ decreases more and more terms are required in the series and hence more and more solutions to (1) must be computed. In many practical applications the Green's function does need to be evaluated in situations where the source and field point are fairly close together and it is in just such cases where the eigenfunction expansion becomes unsuitable. The advantage of the representations which have been derived in this paper is that they appear to work equally well throughout the whole range of physical parameters. Since the choice of $a$ is at our disposal we can always set it to zero whenever $|x|$ is sufficiently large so that we simply recover the eigenfunction expansion. We will thus concentrate on the case of small $|x|$ below. The individual terms in the series for the new representations are of course more difficult to compute than in the eigenfunction expansion and so the trade off between a few complicated terms and a lot of simple terms will determine which method to use for a particular value of $|x|$. Finally it should be noted that very often a large number of calculations are made for the same value of the frequency parameter $K$, in which case the computation of the roots of the dispersion relation becomes less significant.

We will begin with the two-dimensional case. For (53) or (71) to be useful, the 


\begin{tabular}{cccccc}
\hline & \multicolumn{5}{c}{$-(z+\zeta)$} \\
\cline { 2 - 6 }$a$ & 0 & 0.5 & 1 & 1.5 & 2 \\
\hline 0.2 & $2 \times 10^{-45}$ & $3 \times 10^{-70}$ & $3 \times 10^{-100}$ & $2 \times 10^{-135}$ & $1 \times 10^{-176}$ \\
0.3 & $5 \times 10^{-21}$ & $4 \times 10^{-32}$ & $2 \times 10^{-45}$ & $3 \times 10^{-61}$ & $2 \times 10^{-79}$ \\
0.4 & $2 \times 10^{-12}$ & $1 \times 10^{-18}$ & $2 \times 10^{-26}$ & $2 \times 10^{-35}$ & $2 \times 10^{-45}$ \\
0.5 & $2 \times 10^{-8}$ & $2 \times 10^{-12}$ & $3 \times 10^{-17}$ & $5 \times 10^{-23}$ & $1 \times 10^{-29}$ \\
0.6 & $6 \times 10^{-6}$ & $6 \times 10^{-9}$ & $2 \times 10^{-12}$ & $2 \times 10^{-16}$ & $5 \times 10^{-21}$ \\
0.7 & $2 \times 10^{-4}$ & $1 \times 10^{-6}$ & $3 \times 10^{-9}$ & $2 \times 10^{-12}$ & $9 \times 10^{-16}$ \\
0.8 & $1 \times 10^{-3}$ & $3 \times 10^{-5}$ & $2 \times 10^{-7}$ & $1 \times 10^{-9}$ & $2 \times 10^{-12}$ \\
0.9 & $5 \times 10^{-3}$ & $2 \times 10^{-4}$ & $6 \times 10^{-6}$ & $8 \times 10^{-8}$ & $5 \times 10^{-10}$ \\
1.0 & $1 \times 10^{-2}$ & $1 \times 10^{-3}$ & $6 \times 10^{-5}$ & $2 \times 10^{-6}$ & $2 \times 10^{-8}$ \\
\hline
\end{tabular}

Table 1: $4 b^{-2} \mathrm{e}^{-b^{2}}, b=(2-z-\zeta) / a$.

contribution from the neglected terms must be less than the desired accuracy for $G$. These terms are different in the two expressions (53) and (71); the part of $L_{2}$ (given by (52)) that does not depend on $K$ is in effect included in (71). This is

$$
\frac{1}{4 \pi} \sum_{i=1}^{4} E_{1}\left(\frac{x^{2}+\chi_{2, i}^{2}}{a^{2}}\right) .
$$

Now, since $E_{1}(x) \leq x^{-1} \mathrm{e}^{-x}$ and $\chi_{2, i} \geq 2-z-\zeta$, it follows that this term is bounded above (for all $x$ ) by $4 b^{-2} \mathrm{e}^{-b^{2}}$, where $b=(2-z-\zeta) / a$. The other terms that have been neglected in (53) and (71) are of the same or smaller order of magnitude than this one. (The neglected terms do increase with $|K|$ and so for large values of $|K|$ care must be taken in making sure that the neglected terms do not affect the accuracy of the computations; however for values of $K$ of practical interest, $|K|<10$ say, this is not significant.) The value of the bound above is listed in Table 1 for various values of $a$ and $z+\zeta$. It is clear from the table that for a given value of $z+\zeta$, there is an upper bound on the value of $a$ that can be used in (53) or (71). On the other hand, the larger $a$ is the faster the convergence of the series in the expressions for $G$. From the table we can see that the choice

$$
a=\frac{2-z-\zeta}{5}
$$

will ensure the required accuracy, and because this choice is quite conservative it will also take care of any slight increase in the terms due to a large value of $|K|$. Hence, in the results below, we will always take $a$ to be given by (89).

The convergence of the new representation, (53), is shown in Table 2, compared with that of the eigenfunction expansion (34), for $K=1+\mathrm{i}$. When $x=0$, thousands of terms 


\begin{tabular}{|c|c|c|c|c|}
\hline \multirow[b]{2}{*}{$x$} & \multicolumn{2}{|c|}{$\begin{array}{l}z=-0.5 \\
\zeta=-0.2\end{array}$} & \multicolumn{2}{|c|}{$\begin{array}{l}z=-0.01 \\
\zeta=-0.02\end{array}$} \\
\hline & Eqn (34) & Eqn (53) & Eqn (34) & Eqn (53) \\
\hline 0 & & 4 & & 5 \\
\hline 0.05 & 100 & 4 & 100 & 6 \\
\hline 0.1 & 50 & 4 & 50 & 5 \\
\hline 0.2 & 24 & 4 & 20 & 5 \\
\hline 0.5 & 10 & 4 & 10 & 5 \\
\hline
\end{tabular}

Table 2: The number of terms required to achieve 8 decimal place accuracy when $K=1+\mathrm{i}$.

are required to make the eigenfunction expansion converge. The rapid convergence of the representation (53) is clear, as is the insensitivity of the convergence on the values of $x, z$ and $\zeta$. For $z=-0.5, \zeta=-0.2$, the integral representation (33) provides another efficient method for the evaluation of $G$, but for $z=-0.01, \zeta=-0.02$ it does not.

Our numerical computations suggest that there is little to choose between the representations (53) and (71) as methods for computing $G$. The main criterion for choosing between the two is whether one wishes to compute the exponential integrals in (53) or the error functions that are required for the evaluation of $M_{m}$ in (71).

Next we will consider the case when there is a double root to the dispersion relation. The convergence of the new representation, (53) (with the additional contribution $D$ given by (55)), is shown in Table 3, compared with that of the eigenfunction expansion (34), for $K=K_{d}$ and it is clear that the presence of the double root does not significantly affect the convergence characteristics of either the eigenfunction expansion or the new representation. However, our computations suggest that there is little difficulty in computing $G$ accurately on the assumption that all the roots of (1) are simple, even when $K$ is very close to $K_{d}$. In such a case two of the roots are very close together, but provided they are computed to very high accuracy (which is not difficult), the representations (34), (53) and (71) will all produce accurate values for $G$. As an extreme example, we computed $G$ without using the double root formula for a value of $K$ with $\left|K-K_{d}\right| \approx 10^{-10}$. The roots of (1) were computed to 16 decimal digits and the representations (53) and (71) both converged rapidly to a value for $G$ accurate to 5 decimal places.

The convergence characteristics of the new representation, (81), for the three-dimensional Green's function are very similar to those shown above for the two-dimensional case. We will use this representation to exhibit another important property of the new formulas. 


\begin{tabular}{|c|c|c|c|c|}
\hline \multirow[b]{3}{*}{$x$} & \multirow{2}{*}{\multicolumn{2}{|c|}{$\begin{array}{l}z=-0.5 \\
\zeta=-0.2\end{array}$}} & \multirow{2}{*}{\multicolumn{2}{|c|}{$\begin{array}{l}z=-0.01 \\
\zeta=-0.02\end{array}$}} \\
\hline & & & & \\
\hline & Eqn (34) & Eqn (53) & Eqn (34) & Eqn (53) \\
\hline 0 & & 3 & & 4 \\
\hline 0.05 & 85 & 3 & 100 & 5 \\
\hline 0.1 & 45 & 3 & 45 & 4 \\
\hline 0.2 & 21 & 3 & 20 & 4 \\
\hline 0.5 & 7 & 3 & 9 & 4 \\
\hline
\end{tabular}

Table 3: The number of terms required to achieve 8 decimal place accuracy when $K=K_{d}$.

The function $G$, defined by (72), is singular as $r \rightarrow 0$. Indeed

$$
\begin{aligned}
& G \sim-(4 \pi r)^{-1} \quad \text { as } r \rightarrow 0 \quad(\zeta<0) \\
& \sim-(2 \pi r)^{-1} \quad \text { as } r \rightarrow 0 \quad(\zeta=0) .
\end{aligned}
$$

In applications it is important to be able to accurately determine the non-singular part of $G$, since the singular part can be treated analytically. Hence it is usually desirable to have a representation which contains the singularity explicitly. For example the term $-(4 \pi r)^{-1} \operatorname{erfc}(r / a h)$ in $(81)$ can be written as

$$
-\frac{1}{4 \pi r}+\frac{1}{4 \pi r} \operatorname{erf}\left(\frac{r}{a h}\right)
$$

and as $r \rightarrow 0$ the second term tends to a constant. Table 4 shows that the representation (81) (unlike the standard eigenfunction expansion which is singular as $R \rightarrow 0$ ) is sufficiently accurate and computationally efficient for direct computation near to the singular point to be feasible. In the Table we show the value of the regular part of $\operatorname{Re} G$ to 8 decimal places and the truncation parameter required to achieve this accuracy, as the field point approaches the singular point along a horizontal line. Two sets of parameter values are considered: in both cases $K=1+\mathrm{i}$ and the values of $z$ and $\zeta$ are chosen to make the accurate evaluation of $G$ awkward. In the example on the left $z=\zeta=-0.01$ so that $G \sim-1 / 4 \pi R$ as $R \rightarrow 0$ whereas in the example on the right $z=\zeta=0$ so that $G \sim-1 / 2 \pi R$ as $R \rightarrow 0$. The appropriate behaviour is clearly demonstrated, as is the fact that the number of terms required to achieve an accuracy of 8 decimal places in the regular part of $G$ does not increase as we approach the singular point. 


\begin{tabular}{lcccccc}
\hline & \multicolumn{2}{c}{$z=\zeta=-0.01$} & & \multicolumn{2}{c}{$z=\zeta=0$} \\
\cline { 2 - 3 } \cline { 5 - 6 }$R$ & $M$ & $\operatorname{Re} G+(4 \pi R)^{-1}$ & & $M$ & $\operatorname{Re} G+(2 \pi R)^{-1}$ \\
\hline 0.1 & 6 & -0.69267867 & & 6 & 0.07077096 \\
0.01 & 6 & -3.63578886 & & 6 & -0.29143855 \\
0.001 & 5 & -4.05987077 & & 6 & -0.65784498 \\
0.0001 & 5 & -4.06488325 & & 6 & -1.02431195 \\
0.00001 & 5 & -4.06493346 & & 6 & -1.39077971 \\
\hline
\end{tabular}

Table 4: Truncation parameters required to achieve an accuracy of 8 decimal places in the regular part of $G$ using (81), when $K=1+\mathrm{i}$.

\section{CONCLUSION}

In one class of models that is used to describe the interaction of water waves with surfacepiercing permeable structures the flow field inside the structure is described by a harmonic velocity potential that satisfies the standard linear free-surface boundary condition with the complication that the frequency parameter is complex rather than real. In this paper we have considered that accurate evaluation of the Green's function, $G$, in both two and three dimensions, for a situation in which the permeable structure is of constant height and there is no flow through the bottom.

New representations for the Green's function have been obtained using a method developed in I for the standard water wave problem. A one-parameter family of formulas for $G$ has been derived and by varying the parameter the convergence characteristics of our new representations can be altered. Letting $a \rightarrow 0$ results in known eigenfunction expansions.

Results of computations have been presented and these demonstrate the accuracy and efficiency of the new formulas. Other methods are available for the computation of $G$, in particular the Green's function can be represented as an integral and, unlike in the standard water wave problem, there are no poles of the integrand on the real axis and so evaluation of this integral is straightforward. The exception is when the source and field point are both close to the free surface, in which case the integral representation is no longer useful from a computational point of view. The eigenfunction expansion for $G$ represents an efficient method of computation provided the horizontal distance between source and field points is not too small, but when they are close together the expansion becomes very slowly convergent (in the two-dimensional case) or singular (in the threedimensional case). The new representations that have been derived have the advantage that they appear to offer an efficient method for the evaluation of $G$ throughout the fluid 
domain.

\section{APPENDIX}

In this appendix, brief details are given of a method for determining explicitly the complex roots of certain equations $g(w)=0$. An outline of the technique, that includes sufficient information to solve the water-wave dispersion relation, is given below; full details are given in McIver (1998).

\section{Outline of the method}

Suppose that $g(w)=0$ has an infinity of roots (or, equivalently, $g(w)$ has an infinity of zeros) in the complex $w$ plane. First of all it is required to identify a part $\mathcal{D}$ of the complex $w$ plane that contains only a finite number $m$ of the zeros of $g(w)$, and is such that a function $F(z)$ can be constructed with $m$ zeros in the whole complex $z$ plane that can be related to the zeros of $g(w)$ in $\mathcal{D}$. The method then uses complex variable theory to determine a polynomial

$$
P_{m}(z)=\sum_{j=0}^{m} b_{j} z^{j}
$$

whose zeros correspond, both in location and multiplicity, to the zeros of $F(z)$. In the present problem, it is possible to construct an $F$ such that $m=1$ or $m=2$, and thus $P_{m}(z)=0$ can be solved explicitly for $z$. The coefficients $\left\{b_{j} ; j=0, \ldots, m\right\}$ that appear in $P_{m}$ are given by choosing $b_{m}=1$ and solving the $m$ linear equations

$$
\sum_{j=0}^{m}\left[A_{-k-j-1}+I_{k+j}\right] b_{j}=0, \quad k=0, \ldots, m-1,
$$

where

$$
I_{j}=\frac{1}{2 \pi \mathrm{i}} \int_{-1}^{1} \frac{M^{+}(t)-G(t) M^{-}(t)}{X^{+}(t)} t^{j} \mathrm{~d} t
$$

and the coefficients $A_{j}$ and the functions $M^{ \pm}$depend on the particular $F$. The functions $G$ and $X^{+}$are chosen for convenience and are given below.

\section{Distribution of roots}

Consider now the dispersion relation

$$
g(w) \equiv \Gamma+w \tan w=0
$$

where $\Gamma=\alpha+\mathrm{i} \beta$ is a given complex number and $\alpha$ and $\beta$ are non-negative. From the form of $g$, it is clear that for each root $w=w_{0}$ there is a root $w=-w_{0}$, but only 
those roots with Re $w \geq 0$ are needed here. McIver (1998) uses the argument principle of complex-variable theory to show that the number of roots in the strips

$$
R_{n-1}<\operatorname{Re} w<R_{n}
$$

of the complex $w$ plane, where $n$ is an integer and $R_{n}=(2 n+1) \pi / 2$, can be identified as follows. Let $\mathcal{I}_{n}$ be the condition

$$
\tanh \frac{\alpha R_{n}}{\beta}<\frac{R_{n}}{\beta}
$$

and let $\mathcal{J}_{n}$ be the condition

$$
\tanh \frac{\alpha R_{n}}{\beta}>\frac{R_{n}}{\beta}
$$

1. If either $\mathcal{I}_{n-1}$ and $\mathcal{I}_{n}$ or $\mathcal{J}_{n-1}$ and $\mathcal{J}_{n}$ are both true then there is exactly one zero of $g(w)$ for $R_{n-1}<\operatorname{Re} w<R_{n}$.

2. If $\mathcal{I}_{n-1}$ and $\mathcal{J}_{n}$ are both true, then there are no zeros of $g(w)$ for $R_{n-1}<\operatorname{Re} w<R_{n}$.

3. If $\mathcal{J}_{n-1}$ and $\mathcal{I}_{n}$ are both true, then there are exactly two zeros of $g(w)$ for $R_{n-1}<$ $\operatorname{Re} w<R_{n}$.

After the change of variable

$$
w=-\mathrm{i} \Gamma z
$$

equation (93) may be rewritten as

$$
F(z) \equiv z+\frac{1}{2 \Gamma}\left(\log \frac{z-1}{z+1}-2 n \pi \mathrm{i}\right)=0
$$

where the principal branch of the logarithm is to be taken and the branch cut is chosen to be the interval $(-1,1)$ on the real axis. For each $n$, the zeros of $F(z)$ in the whole cut $z$ plane correspond to the zeros of $g(w)$ in the strip (94).

\section{Explicit calculation of the roots}

The algorithm may be summarized as follows. For a given $\Gamma=\alpha+\mathrm{i} \beta$, the number of roots of (93) in each strip $R_{n-1}<\operatorname{Re} w<R_{n}, n=0,1,2, \ldots$, is determined from the inequalities in section 6. Explicit formulae for these roots are found by taking $G(t)=-1$,

$$
\begin{gathered}
X^{+}(t)=1 /\left[\mathrm{i}\left(1-t^{2}\right)^{1 / 2}\right], \\
M^{ \pm}(t)=\left[t+\frac{1}{2 \Gamma}\left(\log \frac{1-t}{1+t}-(2 n \mp 1) \pi \mathrm{i}\right)\right]^{-1},
\end{gathered}
$$




$$
\begin{aligned}
& A_{-1}=\frac{\mathrm{i} n \pi}{\Gamma}, \\
& A_{-2}=\frac{1}{2 \Gamma^{2}}\left[2 \Gamma-\Gamma^{2}-2 n^{2} \pi^{2}\right], \\
& A_{-3}=-\frac{\mathrm{i} n \pi}{2 \Gamma^{3}}\left[2 n^{2} \pi^{2}+\Gamma^{2}-4 \Gamma\right], \\
& A_{-4}=\frac{1}{24 \Gamma^{4}}\left[24 n^{4} \pi^{4}+12 n^{2} \pi^{2} \Gamma(\Gamma-6)+\Gamma^{2}\left(24-4 \Gamma-3 \Gamma^{2}\right)\right],
\end{aligned}
$$

and then solving the system (91) to determine $\left\{b_{j} ; j=0, \ldots, m-1\right\}$. (Note that the integrals $I_{j}$ must be calculated numerically.) The zeros of $P_{m}(z)$ are then calculated and finally (97) is used to transform these to zeros of $g(w)$.

\section{Double roots}

Double roots of $g(w)=0$ occur at isolated values of $\Gamma=-w \tan w$ when $w$ is a roots of

$$
\sin 2 w+2 w=0 .
$$

Explicit formulae for these values of $w$ may be found by the above method (see McIver 1998), however numerical values only are given here in Table 5 for the five roots within the fourth quadrant that have smallest modulus. It should be noted that if $w=w_{0}$ is a root of (105), then so is $-w_{0}$ and $\pm \bar{w}_{0}$.

\begin{tabular}{cc}
\hline$w / 2$ & $\Gamma$ \\
\hline $4.21239-2.25073 \mathrm{i}$ & $1.65061+2.05998 \mathrm{i}$ \\
$10.7125-3.10315 \mathrm{i}$ & $2.05785+5.33471 \mathrm{i}$ \\
$17.0734-3.55109 \mathrm{i}$ & $2.27847+8.52264 \mathrm{i}$ \\
$23.3984-3.85881 \mathrm{i}$ & $2.43112+11.6888 \mathrm{i}$ \\
$29.7081-4.09370 \mathrm{i}$ & $2.54799+14.8458 \mathrm{i}$ \\
\hline
\end{tabular}

Table 5: Values of $\Gamma$ for which there are double roots of the dispersion relation (93) together with the corresponding roots $w$ of (105).

\section{APPENDIX. REFERENCES}

Abramowitz, M., \& Stegun, I. A. (1965). Handbook of Mathematical Functions. Dover, New York.

Carslaw, H. S., \& Jaeger, J. C. (1959). Conduction of Heat in Solids (2nd ed.). Oxford University Press. 
Chaudhry, M. A., Temme, N. M., \& Veling, E. J. M. (1996). Asymptotics and closed form of a generalized incomplete gamma function. J. Comput. Appl. Math., 67, $371-379$.

Dalrymple, Robert A., Losada, Miguel A., \& Martin, P. A. (1991). Reflection and transmission from porous structures under oblique wave attack. J. Fluid Mech., 224, 625-644.

Ijima, T., Chou, C. R., \& Yumura, Y. (1974). Wave scattering by permeable and impermeable breakwater of arbitrary shape. In Proc. 14th Intl Conference on Coastal Engineering, ASCE, pp. 1886-1905.

Linton, C. M. (1999). Rapidly convergent representations for Green's functions for Laplace's equation. Proc. Roy. Soc. Lond., A. To appear.

Lozier, D. W., \& Olver, F. W. J. (1995). Numerical evaluation of special functions. In W. Gautschi (Ed.), Mathematics of Computation 1943-1993: A half-century of computational mathematics. American Mathematical Society.

McIver, P. (1998). The dispersion relation and eigenfunction expansions for water waves in a porous structure. J. Engng. Math., 34, 319-334.

MCLean, N. (1999). Water wave diffraction by segmented permeable breakwaters. $\mathrm{Ph} . \mathrm{D}$. thesis, Loughborough University. In preparation.

Sollitt, C. K., \& Cross, R. H. (1972). Wave transmission through permeable breakwaters. In Proc. 13th Intl Conf. on Coastal Engineering, ASCE, pp. 1827-1846.

Strain, John (1992). Fast potential theory. II. Layer potentials and discrete sums. J. Comput. Phys., 99, 251-270.

Sulisz, W. (1985). Wave reflection and transmission at permeable breakwaters of arbitrary cross-section. Coastal Eng., 9, 371-386.

Yu, X., \& Chwang, A. T. (1994). Wave motion through porous structures. J. Engrg. Mech., 120, 989-1008. 